\title{
Node Density Estimation in VANETs Using Received Signal Power
}

\author{
Golnar KHOMAMI ${ }^{1}$, Prakash VEERARAGHAVAN ${ }^{1}$, Fernando Pérez FONTÁN ${ }^{2}$ \\ ${ }^{1}$ Dept. of Computer Science and Computer Engineering, La Trobe University, Bundoora, Victoria, Australia \\ ${ }^{2}$ Dept. of Signal Theory and Communications, University of Vigo, Spain \\ g.khomami@latrobe.edu.au,p.veera@latrobe.edu.au, fpfontan@tsc.uvigo.es
}

\begin{abstract}
Accurately estimating node density in Vehicular Ad hoc Networks, VANETs, is a challenging and crucial task. Various approaches exist, yet none takes advantage of physical layer parameters in a distributed fashion. This paper describes a framework that allows individual nodes to estimate the node density of their surrounding network independent of beacon messages and other infrastructurebased information. The proposal relies on three factors: 1) a discrete event simulator to estimate the average number of nodes transmitting simultaneously; 2) a realistic channel model for VANETs environment; and 3) a node density estimation technique. This work provides every vehicle on the road with two equations indicating the relation between 1) received signal strength versus simultaneously transmitting nodes, and 2) simultaneously transmitting nodes versus node density. Access to these equations enables individual nodes to estimate their real-time surrounding node density. The system is designed to work for the most complicated scenarios where nodes have no information about the topology of the network and, accordingly, the results indicate that the system is reasonably reliable and accurate. The outcome of this work has various applications and can be used for any protocol that is affected by node density.
\end{abstract}

\section{Keywords}

Keywords: VANETs, node density, broadcast storm problem, safety messages, channel model, RSS

\section{Introduction}

Predicting node density is an important problem in any network topology. The node density may be used as a parameter to predict the congestion in the network. The congestion may be due to the application layer traffic and the networkmaintenance traffic that are mostly broadcast in nature.

In an infrastructure network (both wired and wireless), estimating the node density is a simple job, and the node density can easily be restricted to a maximum threshold value. Hence, the delay-jitter due to possible congestion can be predicted. However, in an infrastructure-less network, node density prediction is still a challenging open problem.

A Mobile Ad hoc network (MANET) is an autonomous collection of mobile users that communicate over relatively bandwidth constrained wireless links. Since the nodes are mobile, the network topology may change rapidly and unpredictably over time. A VANET is a special type of MANET where the nodes forming the network are the vehicles on the road. VANETs offer greater challenges than MANETs due to the higher mobility of nodes and the realtime requirements for the application layer packets. A Dedicated Short Range Communication (DSRC) based VANET provides an opportunity to enable communication between cooperative safety systems in order to decrease road traumas and to improve traffic efficiency. VANETs provide drivers with the capability to improve their awareness of their surroundings. This is achieved through the sensors attached to the vehicles forming an ad hoc network and communicating wirelessly with surrounding vehicles to warn each other of changing road conditions or dangers ahead on the road to avoid accidents. This is called Cooperative Collision Avoidance (CCA) or Safety Applications.

\subsection{Motivation}

Vehicle to Vehicle (V-2-V) safety applications have a strict real-time requirement of $100 \mathrm{~ms}$ application to application latency and a Packet Delivery Ratio (PDR) of at least $90 \%$. The conventional IEEE 802.11 protocols may not meet these requirements. It has been shown in the literature that only if a neighbourhood density is low or if there is a small number of nodes contending for the channel to broadcast their messages, then a PDR of $90 \%$ or more can be achieved for a particular transmitting node. However, if there are more nodes attempting to broadcast simultaneously, this will result in a collision and thus will reduce the PDR. This effect is even worse in very dense networks, resulting in the well-known broadcast storm effect [1]. In addition, maintaining the connectivity in disconnected and sparse networks is another challenge in $\mathrm{V}-2-\mathrm{V}$ communications and requires awareness of the individual nodes about their surroundings. Several researchers have proposed topology control solutions to address the above mentioned problems. In most of these methods beacon messages are used to estimate network density. Beacon messages are small messages containing the 
information for individual nodes to become aware of their surroundings. However, in dense networks, any extra transmission could worsen the highly congested channel and in the case of broadcast storm and sparse networks the beacon messages might not be received. Therefore, relying only on beacon messages for estimating node density can make the system unreliable. Since an accurate node density estimation technique can have a strong influence on improving the network throughput, we propose one such technique implemented in a distributed fashion and totally independent of beacon messages.

\subsection{Solution Outline}

In this section we explain the proposed solution which consists of three stages. Briefly, stages include i) calculation of the number of nodes transmitting simultaneously, ii) implementing a realistic channel model for the vehicular communication environment, and iii) the process of the node density estimation. Details of the three stages are as follows.

Stage 1: In the first stage, we performed a discrete event simulation to estimate the average number of nodes transmitting simultaneously, when there are $n$-nodes contending for a shared channel. At the end of this stage, an equation showing the relation between number of contending nodes and average number of successful nodes transmitting simultaneously was constructed.

Stage 2: During the second stage, we implemented a realistic channel model for the vehicular network. Using our channel model, $r$-reference nodes will be transmitting a frame simultaneously. A reference receiver will measure the sum of all received signal powers and will form another equation showing the relation between RSS, and number of simultaneously transmitting nodes. Note that the overall received signal is subjected to complex valued fading. The overall resulting signal will be the coherent (amplitude and phase) sum of all received signals in complex form.

Stage 3: The third stage is the estimation process. At this stage we assume that the two equations from the above two stages are available to every vehicle in the VANET. In this case, every vehicle will collect values of received RF signal power. If the Carrier-to-Interference plus Noise Ratio (CINR) is sufficiently large and is within the acceptable threshold value, this means that exactly one vehicle will be transmitting the frame. Otherwise, it means a collision has happened, we consider a collision as interference. Then based on the equations constructed in Stage 2, the vehicles will estimate the average number of nodes that transmitted simultaneously. Finally, using the equation constructed in Stage 1 , a vehicle can estimate the node density. Figure 1 demonstrates the flowchart of our proposed node density estimation procedure and how it can be used to prevent a problem such as broadcast storm problem.

The rest of this paper is organized as follows. The next section presents the background of the domain including node density and its related works, the channel access procedure and an introduction to channel models in VANETs. In Section 3, the methodology of the proposed node density estimation process is explained in detail, containing an explanation on the mentioned three stages of our proposal and simulation setups. This will be followed by a discussion on evaluating accuracy and consistency of the system in Section 4. Finally, in Section 5, the conclusion is followed by an enumeration of open questions, limitations and future direction.

\section{Background and Related Work}

\subsection{Node Density Estimation}

Node density estimation is a key challenge in most of the solutions proposed in the literature to reduce collisions and address the connectivity problem in sparse networks. In an infrastructure based network, node density is known through the access point. In the V-2-V environment different approaches are used to detect node density. In the following, property of different solutions will be discussed. However, the discussion about strength and weak point of each solution and a feature-based comparison of these solutions with our proposal will be discuss in a separate section at the end of the paper.

Using the regular beacon messages, each node is able to get some idea about their neighborhood and determine their surrounding node density. For instance in [2], [3] a distributed network density estimation technique is introduced. In this scheme, a road segment is divided into multiple cells. The density of the network in each cell can be calculated by a designated vehicle called the "group leader". The average density of the network will be calculated by communication between group leader vehicles of all cells. In [4], the traffic condition is evaluated in terms of average velocity for traffic segments. The vehicles periodically exchange and update their current position and traffic information (average velocity) for surrounding vehicles. The effectiveness of these algorithms greatly depends on the communication between their group leaders and how this information is disseminated to individual nodes. Also, it is essential that the beacon messages are successfully received by the group leaders to make a realistic estimation. However, in the event of a broadcast storm, even the beacon messages may not be received successfully. This may reduce the accuracy of the detection algorithm. In a rather different approach, in [5], node density is detected by analysing the sequence number of the consecutive received beacon messages. In this solution, any loss of beacon messages will be assumed as collision.

Node density may also be estimated through other means. In [6], [7], the vehicle density is estimated based on the traffic flow theory. In [6] node density is calculated from the average fraction of time that the vehicle is stopped or slowed down. Nevertheless, this system presents low per- 


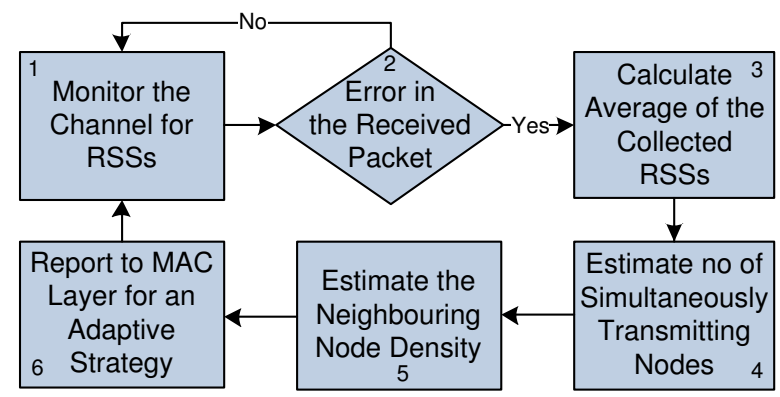

Fig. 1. Flowchart of density prediction and cross layer adaptation.

formance when vehicles are in free flow state. The solution presented in [7] estimates node density based on the two parameters of velocity and acceleration of vehicles. It is showed that in the solution based on speed and acceleration, node density can be estimated at the earlier stage than in the solution presented in [6]. Still both solutions are not accurate in sparse networks.

Lack of an accurate estimation technique, capable of working in chaotic network conditions motivated us to propose our solution. Our proposal is not relying on any exchanged information between nodes therefore, can perform well in the situations with high packet loss due to collision. It also can perform well in sparse networks as well as in dense networks.

\subsection{Channel Access in VANETs}

Choosing a suitable communication protocol for the VANET environment is important. Distributed Coordinated Function (DCF) is a Carrier Sense Multiple Access (CSMA) protocol that was originally developed for wireless LANs. It has been adapted by IEEE Standards for DSRC. DCF is a suitable candidate for $\mathrm{V}-2-\mathrm{V}$ communications due to its decentralized operation, adaptation to various traffic loads, dynamic reconfiguration when there is a change in the network topology and the support for both unicast and broadcast transmissions

In 802.11 based MAC protocols, DCF uses carrier sensing to determine the availability of the shared channel prior to transmission. Two different types of carrier sensing are supported by DCF: Virtual Carrier Sensing (VCS) and Physical Carrier Sensing (PCS). VCS is used for unicast traffic in which each node sends the Request to Send (RTS)/Clear to Send (CTS) to reserve the access to the channel prior to transmission. However, a channel reservation mechanism using RTS/CTS adds latency and overhead and therefore does not scale well to broadcast traffic which is used for time-critical applications like CCA. In PCS the physical layer is responsible for sensing the channel and finding if the medium is busy. Broadcast transmission only uses PCS and does not require the establishment of an association between the stations before data transmission and thus does not incur extra overhead due to its simple operation. However, the broadcast mode lacks reliability. In the absence of an acknowledgement, there is no means for the transmitting node to know whether the packet is received successfully by all its neighbouring nodes. Also in the absence of a retransmission, the Contention Window (CNWD) size is always kept constant to its minimum value, and the transmission happens at the lowest possible data rate.

In 802.11 DCF, when a station has a frame to broadcast and it determines that the medium is idle for a period equal or greater than one Distributed Inter Frame Space (DIFS), the station transmits the frame immediately. If the medium is found to be busy, then the transmission will not happen immediately and the backoff algorithm is used.

The backoff algorithm works in a slotted time mode. There is a Backoff Counter $(B C)$ that contains certain values between zero and the maximum value of CNDW. In $802.11 \mathrm{p}$ networks, the CNDW size for broadcast transmission is 16 . If one slot has passed with the channel being idle, the value of $B C$ is decremented by one. If the channel is not idle, then the station will freeze the contention timer until the channel is free for a period equal to one DIFS. The value of $B C$ is decremented until it reaches zero. As soon as the $B C$ reaches zero, the frame is transmitted, provided that the channel is still idle. In an unsaturated condition, where the channel is free most of the time, a node can transmit a frame as soon as it is ready in the transmit buffer and thus, the backoff algorithm is rarely invoked. However, in a saturated condition the backoff algorithm will be invoked for every single frame to be transmitted. If two or more nodes happen to choose the same $B C$ value in a distributed fashion, it is expected that all these nodes will transmit simultaneously, resulting in a collision. Figure 2 illustrates the basic 802.11 MAC channel access procedure based on DCF.

Next section will give an introduction to channel models for VANETs followed by an overview of related works available in the literature and the reason of choosing the selected channel model.

\subsection{Channel Model for VANETs Environment}

To evaluate the performance of our proposed node density detection technique, we implemented a channel model to simulate and observe the behavior of the received signal strength for different node densities. Selecting an accurate channel model is crucial in order not to induce errors 


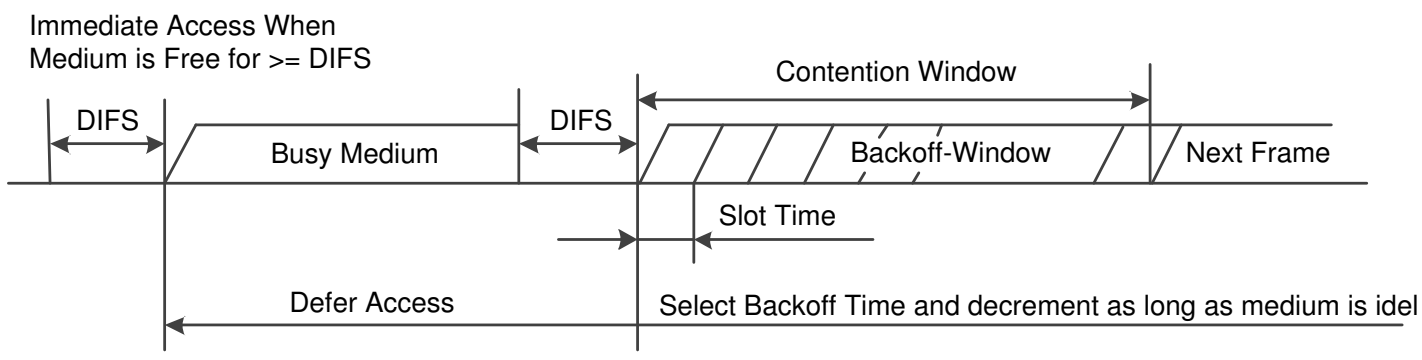

Fig. 2. Basic IEEE 802.11 distributed coordination function access procedure [8].

in the node density detection process in real-time scenarios which, in turn, would also be misleading in the design and performance evaluation of the higher layers. Channel model refers to the impact of the propagation environment (channel) on the radio signal. As a radio signal propagates through space, it may be reflected, scattered or diffracted on the different features in the environment. Here we concentrate on its effects on the received signal power (fading) assuming narrowband conditions with no significant delay spread. Many models are available for cellular systems. However, VANETs operate in a slightly higher frequency band, 5.9 GHz. Moreover, the height of the transmit and receive antennas are similar and very close to the ground, unlike the cellular case where base stations are usually set on elevated sites. The difference between VANETs and conventional WLANs operating outdoors is that, although they operate in similar frequency bands, their terminal mobility patterns are completely different. In case of V-2-V communications the channel fluctuations may be way faster [9].

\section{Existing Channel Models}

In general, there are three fundamental approaches to channel modelling: deterministic, stochastic and geometry based stochastic (also called physical statistical models). The deterministic approach computes the realization of the propagation channel in a specific location and environment. A deterministic channel model uses Maxwell's equations or simplifications thereof to determine the field strength at all points and times. This kind of approach can be very realistic and accurate but it needs extended computation times and needs an exact knowledge of the boundary conditions for simulating specific scenarios in the form of terrain and building databases [10]. In the stochastic approach the distributions of the various propagation characteristics are modelled for generic rather than site specific scenarios. Stochastic channel models work much faster than sitespecific ones. Still, there is the need to derive the parameters for such models empirically from extensive measurements. The geometry-based stochastic approach usually provides a much comprehensive insight into the channel behavior as it resembles a site-specific model where the scenario layout is taken into consideration but still, it refers to a generic scenario. Such models are more suitable for MIMO or diversity configurations where angles of arrival and departure play an important role. In the literature numerous models are available, mostly following the stochastic and the geometric ap- proaches. Extensive overviews of these models can be found in [11] and [12]. We have picked the stochastic approach given its simplicity while maintaining good accuracy levels.

\section{A Realistic Channel Model for VANETs}

In general, the received signal is affected by three separate processes: path loss, large scale or shadow fading, and small scale or multipath fading [13]. Understanding and considering all of these effects is equally important when developing a realistic channel model. The path loss (PL) determines the local average received signal power and depends on the distance between transmitter and receiver. The shadowing term $(\mathrm{SH})$ takes into account the effect of the surrounding obstacles, thus modifying the path loss value. The signal can reach the receiver through multiple paths bouncing on the ground, walls, trees, other cars, etc. Given that the relative phases of these contributions can drastically change with small position changes of Tx, Rx or the scatterers, this gives rise to small scale-fading or multipath (MP) fading. We will make the assumption that the instantaneous received signal is subjected to shadow fading whose changes will be so small between consecutive RSS measurements that can be considered constant, especially in traffic jam conditions. Moreover, the multipath induced variations will be considered to be uncorrelated between consecutive RSS measurements. The propagation conditions in VANETs can roughly be classified into three states: Line-of-Sight (LOS), NonLine-of-Sight (NLOS) due to large obstacles such as buildings, and Obstructed-Line-of-Sight (OLOS), especially due to other vehicles. According to [14], highway scenarios can be described by LOS and OLOS conditions while the NLOS case can be neglected. Including OLOS paths, which is often disregarded in $\mathrm{V}-2-\mathrm{V}$ studies, adds enhanced realism to the simulation results. The impact of obstructing vehicles has been studied in detail by Abbas et al. in [15]. Our simulations employ such a channel model which is based on the work reported in [15].

\section{Methodology}

\subsection{Stage1: Estimating the Number of Simultaneously Transmitting Nodes}

In first stage of our proposal, we developed a discrete 


\begin{tabular}{|c|c|c|c|c|}
\hline \multicolumn{3}{|c|}{ Coefficients (with $95 \%$ confidence bounds) } & \multicolumn{2}{|c|}{ Goodness of fit } \\
\hline $\mathrm{a}$ & 0.0293 & $(0.02722,0.03137)$ & SSE & 1.892 \\
\hline $\mathrm{b}$ & 1.13 & $(1.117,1.143)$ & R-Square & 0.9992 \\
\hline $\mathrm{c}$ & 0.8934 & $(0.8442,0.9427)$ & Adjusted R-Square & 0.9992 \\
\hline & & & RMSE & 0.09799 \\
\hline
\end{tabular}

Tab. 1. Coefficients and goodness of fit for fitted power equation 1 .
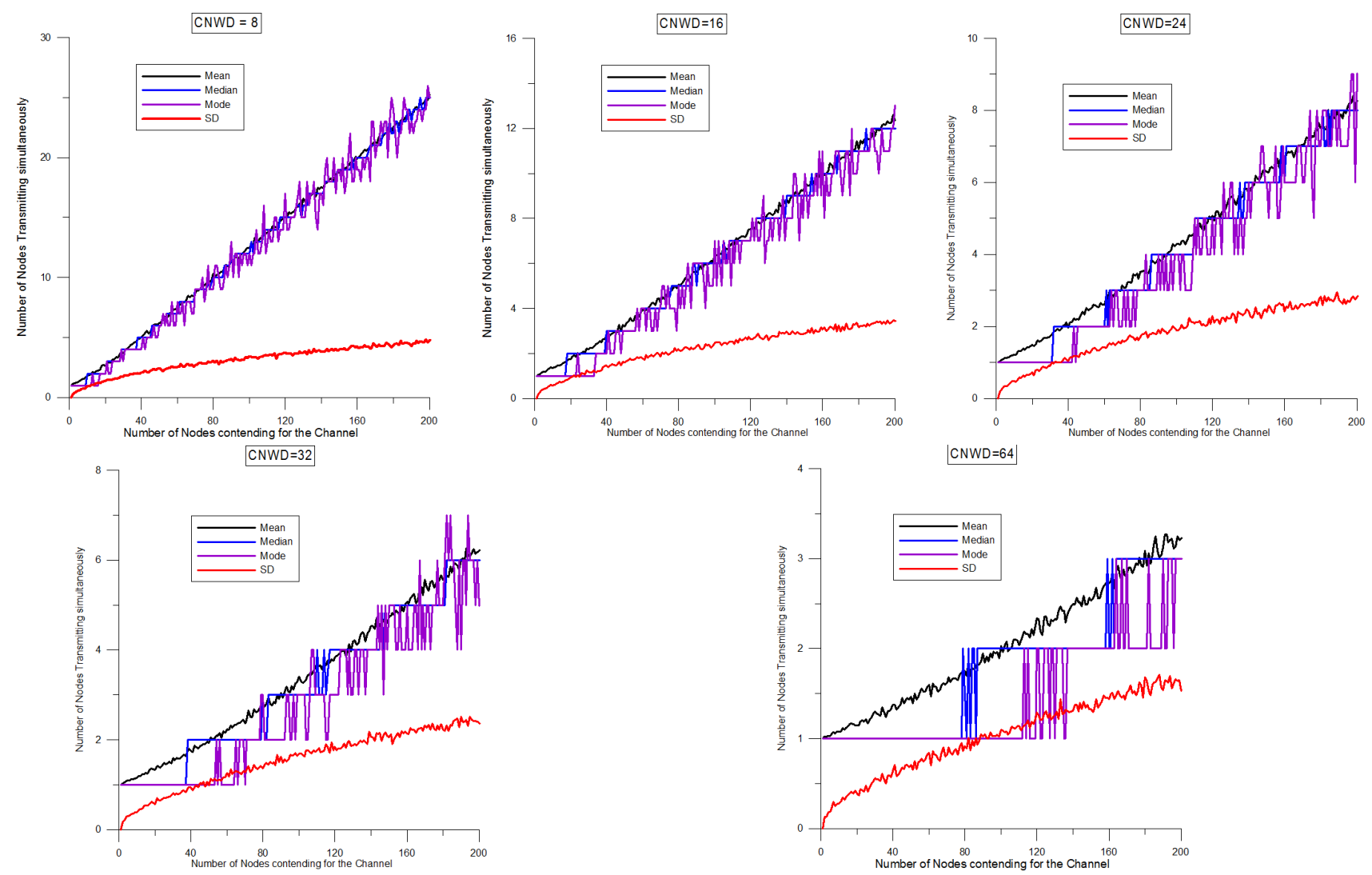

Fig. 3. Average, median, mode and standard deviation of the number of nodes transmitting simultaneously with different $C N W D$ size from 8 to 64 .

event simulator to implement the backoff process in five experiments for different CNWD size 8, 16, 24, 32 and 64. In each experiment, at a given instant of time, there will be $n$ nodes contending for a channel (number of nodes varies from 1 to 200 nodes). For each simulation with a certain number of nodes, each node will choose a random backoff counter value $B C_{i}$. The nodes that choose the smallest $B C$ value will transmit simultaneously. While the nodes are waiting for their $B C$ to reach zero, new nodes might join the queue. The number of new nodes joining the queue depends on the inter-arrival rate. In our simulation, we consider the same inter-arrival time for every node in a neighbourhood, as we are considering a snapshot of an emergency situation. Each experiment is run 500 times to remove any simulation artefacts. At the end of the 500th run, the mean, median, mode and standard deviation of the number of nodes transmitting simultaneously, is computed. The results are presented in Fig. 3.

In all simulations, it can be observed that the mean, median and the mode are close to each other. This shows that the obtained results are consistent and reliable. Also the results are evenly distributed around the mean. Since the standard deviation is small, it can be inferred that the data dispersion from the mean is also small. Thus the data are close to the mean. When $C N W D$ is 32 or 64, the data dispersion from the mean is small compared with the case where $C N W D$ is 8 or 16 . When $C N W D$ is 24 , the dispersion is moderate. As expected, the median is like a "step-function".

In transmission of VANET Safety Messages, CNWD is always set at 16 (from zero to 15). Since in this work the interest is in broadcast transmissions the results of $C N W D=16$ were used and a curve was fitted with $95 \%$ confidence bounds through the mean data obtained in the simulation. Equation (1) shows the relation between the number of contending nodes (Nodes) and the number of successful nodes to transmit simultaneously $(N)$.

$$
N=a \times \text { Nodes }^{b}+c
$$

The coefficients $a, b, c$ and the goodness of fit test results are listed in Tab. 1. Figure 4 shows the comparison between the 


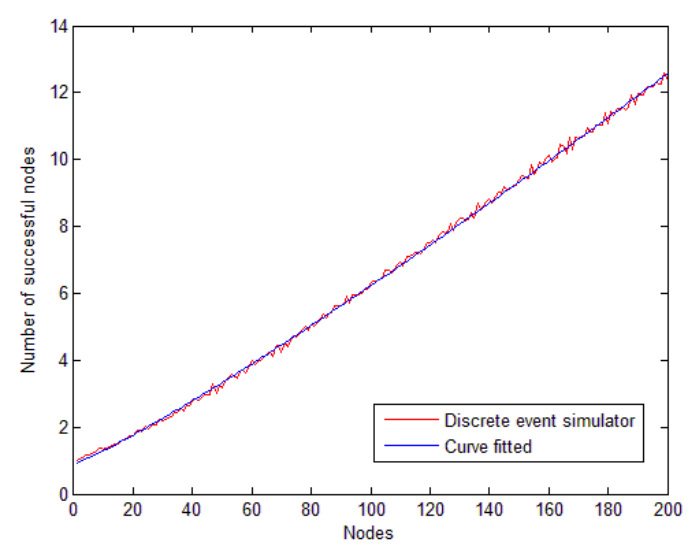

Fig. 4. Results of curve fitting of discrete event simulator.

results obtained through simulation and the results obtained through (1).

\subsection{Stage 2: VANET Channel Model and Simulation Setups}

A realistic statistic channel model was proposed in [15]. This model is a stochastic one including path loss, shadowing and multipath effects. Its parameters have been obtained empirically from measurements perfumed in urban and highway environments. We have selected this model given its simplicity while it still provides and excellent accuracy in reproducing the received signal level. The most sensitive issue faced here in addition to a good characterization of the received power, is the kind of Doppler effects to be expected. This has an impact on the rate of change to be expected due to multipath. We will assume that consecutive signal samples of the the multipath component will be uncorrelated regardless of the general mobility conditions, even in traffic jams. However, shadow fading will be considered to be constant throughout the process of estimating the RSS by each of the nodes which involves a number of consecutive RSS readings as discussed later on.

Parameter RSS ( $\mathrm{dBm})$ can be calculated as a function of the transmit power, $\mathrm{Pt}$, the different propagation factors (PL, SH and MP) and the node antenna gains (GTx and GRx) using (2).

$$
R S S=P t-P L-S H-M P+G T x+G R x .
$$

In general PL $(\mathrm{dB})$ is calculated using (3) which is a log-distance power law model [10] where $d$ is the distance between $T X$ and $R X, n$ is the path loss exponent and $P L_{0}(\mathrm{~dB})$ is path loss at a reference distance $d_{0}(\mathrm{~m}) . X_{\sigma}$ represents the shadowing component which is characterized by a zero-mean Gaussian distributed random variable with standard deviation $(\sigma)$.

$$
P L(d)=P L_{0}+10 n \log _{10}\left(\frac{d}{d_{0}}\right)+X_{\sigma}
$$

Although (3) is widely used as a reference model for vehicular networks, measured data in [15] showed that the dual-slope model proposed in [16] is more accurate. Equation (4) assumes that the $P L$ decays with exponents $n_{1}$ and standard deviation $\sigma$ for distances up to $d_{b}$ and from there on, it decays with path loss exponents $n_{2}$ and standard deviation $\sigma$.

$$
P L(d)=\left\{\begin{array}{cl}
P L_{o}+10 n_{1} \log _{10}\left(\frac{d}{d_{0}}\right)+X_{\sigma}, & \text { if } d_{0} \leq d \leq d_{b} \\
P L_{o}+10 n_{1} \log _{10}\left(\frac{d_{b}}{d_{0}}\right)+ & \text { if } d>d_{b} \\
10 n_{2} \log _{10}\left(\frac{d}{d_{0}}\right)+X_{\sigma}, &
\end{array}\right.
$$

Distance $d_{b}$ is normally calculated using a flat earth model which assumes that the received signal is made up of a direct and reflected ray. Distance $d_{b}$ corresponds to the point where the phase difference between the two rays is $\frac{\pi}{2}$, which is equivalent to the reflected ray path length being equal to $d_{b}+\frac{\lambda}{4}$, where $\lambda$ is the carrier's wavelength. This model supposes that the reflected ray is affected by a reflection coefficient equal to -1 . For distances up to $d_{b}$, the phase difference changes significantly with the link distance, giving rise to consecutive signal enhancements and cancellations; as the distance increases beyond $d_{b}$, the phase difference is too small and the two signals interfere destructively.

Instead of determining $d_{b}$ in a deterministic way, this parameter was found to be 104 meters from the analysis of the experimental data [15], [16]. The other parameters of Dual-Slope Path Loss model (4) are summarized in Tab. 2. We concentrated on the analysis of highway scenarios. The measurements in [15] showed that NLOS conditions mainly happen in urban scenarios, and that, for highway scenarios a two-state model (LOS-OLOS) is sufficiently accurate.

The probabilities of being in the LOS or OLOS state were determined empirically and in [15] a distance dependent plot is provided. In our simulations, the decision as to whether each of the signals coming from the transmitting nodes are in LOS or OLOS is made based on such distancedependent probability. The curve in [15] was fitted with an exponential function shown in Fig. 5. Finally, the synthesis of fast-fading samples was done by means of two complex Gaussian random number generators of mean zero and variance $1 / 2$ in phase quadrature. Taking the absolute value of the resulting complex number yields a Rayleigh distributed series with normalized power equal to one. All signal transmissions present at the receiver are finally combined to calculate the RSS parameter. The overall resulting value will be the coherent (amplitude and phase) sum of all received signals in complex form. The complex component comes from the multipath part of the model.

\begin{tabular}{|c|c|c|c|c|}
\hline & $n_{1}$ & $n_{2}$ & $P L_{0}$ & $\sigma$ \\
\hline \hline LOS & -1.66 & -2.88 & -66.1 & 3.95 \\
\hline OLOS & - & -3.18 & -76.1 & 6.12 \\
\hline
\end{tabular}

Tab. 2. Parameters for the dual-slope path loss model for highway scenario [15]. 
Other Simulation Setups The scenarios simulated correspond to a 300 meter long section of highway with 4 lanes on each direction (8 lanes in total). The vehicle positions are drawn randomly using uniform distributions. The random locations of the roadside is taken into account by the propagation model. The LOS/NLOS state is also determined by random draws, as discussed above. Figure 6 shows the vehicle positions in a sample scenario with 15 transmitting and one receiving node. In the simulations, the antenna gains were set to $3 \mathrm{dBi}$ and transmit power $(P t)$ to $20 \mathrm{dBm}$. The transmit data rate was set to $6 \mathrm{Mbps}$, the fixed value for broadcasting safety masses in IEEE802.11p.

\subsection{Stage 3: Estimating Number of Simultaneously Transmitting Nodes Using RSS}

In order to evaluate the impact of node density on RSS different scenarios were simulated with different numbers of simultaneously transmitting nodes and the combined RSS was calculated. Figure 7 shows the calculated overall RSS in scenarios with different numbers of simultaneously transmitted signals. As can be seen, the higher the number of transmitting nodes, the higher the value of the resulting RSSs. In other words, the RSS increases as the node density increases. However, the fluctuation in the graph is related to the positioning of the nodes and the distance of the closest nodes to the receiving node. It has been observed that if one node is placed very close to the $R X$ node its signal strength can be dominating in the calculation of the sum of all RSSs. This fluctuation in the collected results or, in other words, the impact of the node positioning, can be misleading in the overall calculation of the number of simultaneously transmitting nodes based on RSS. Therefore, in order to observe the impact of RSS in node density in a more accurate way, the simulation of each scenario was performed once for 20 times, 50 times and 500 times and the average of the collected value was calculated. Figure 8 shows how the fluctuation in the collected results reduces and the curve becomes

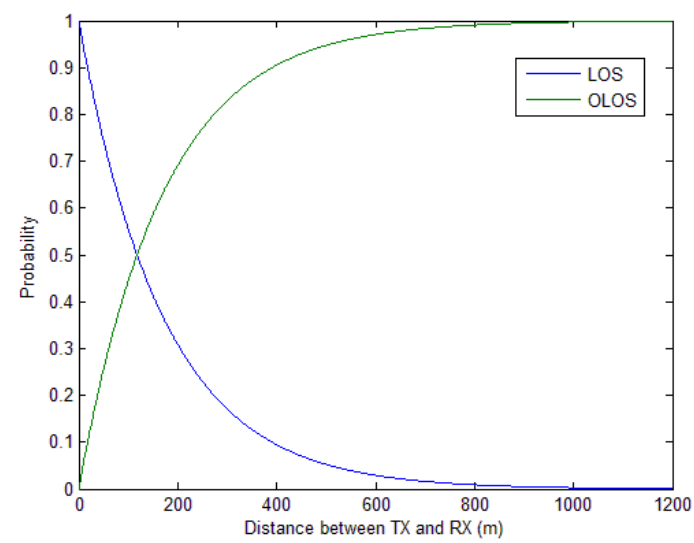

Fig. 5. The probabilities of being in LOS and OLOS, respectively, depending on distance between TX and RX (exponential curve fitted to the curve provided in [15].

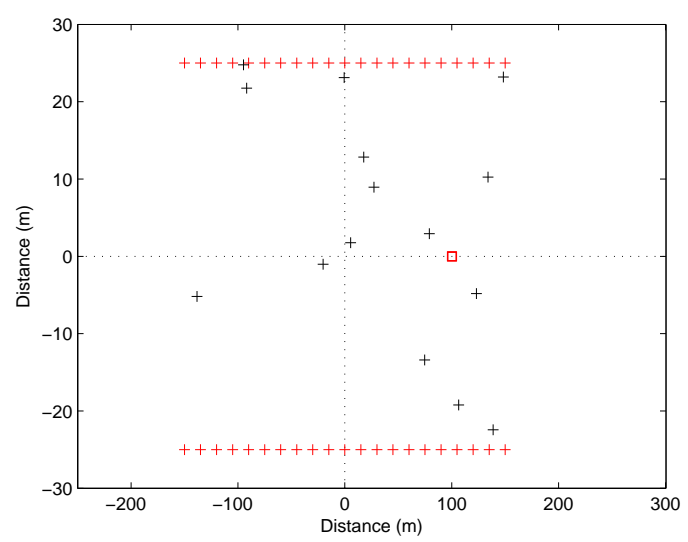

Fig. 6. Example of a scenario with 15 transmitting (black plus signs) and one receiving node (red square).

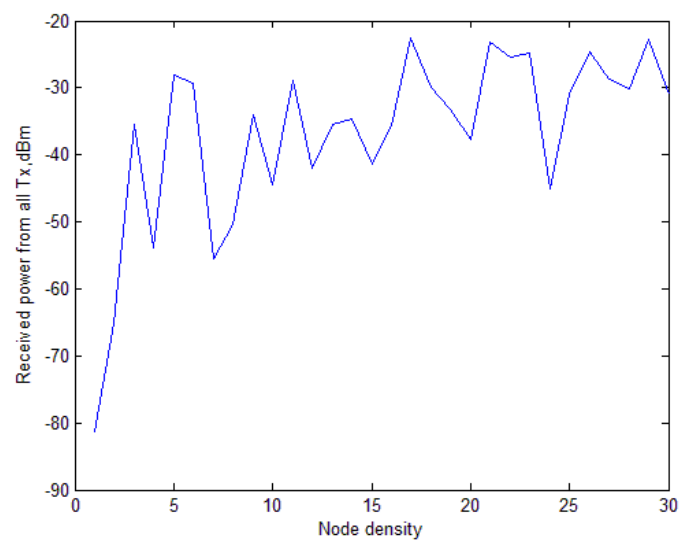

Fig. 7. RSS in scenarios with different numbers of transmitting nodes.

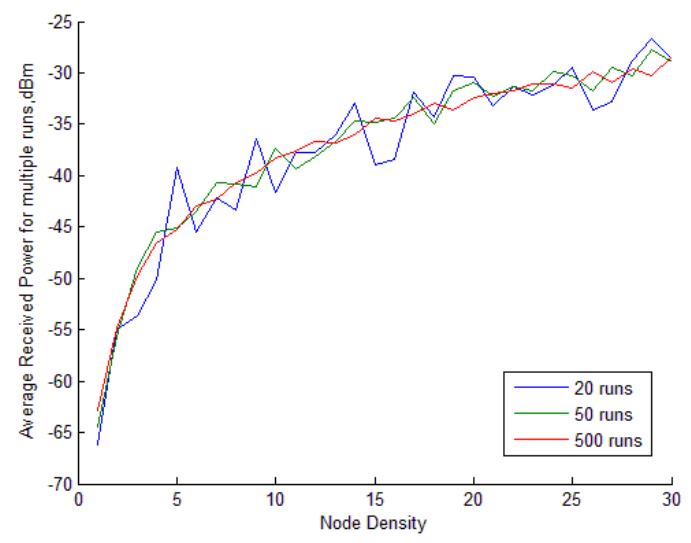

Fig. 8. Average of 500 runs of all scenarios. 


\begin{tabular}{|c|cc|c|c|}
\hline \multicolumn{2}{|c|}{ Coefficients (with 95\% confidence bounds) } & \multicolumn{2}{c|}{ Goodness of fit } \\
\hline \hline$d$ & -26.6 & $(-27.82,-25.39)$ & SSE & 3.099 \\
\hline$e$ & -0.3312 & $(-0.3644,-0.2981)$ & R-Square & 0.9982 \\
\hline$f$ & -40.94 & $(-41.85,-40.04)$ & Adjusted R-Square & 0.998 \\
\hline$g$ & -0.01387 & $(-0.01385,-0.01181)$ & RMSE & 0.3452 \\
\hline
\end{tabular}

Tab. 3. Coefficients and goodness of fit for fitted exponential equation (5).

\begin{tabular}{|c|c|c|c|c|}
\hline \multicolumn{3}{|c|}{ Coefficients (with 95\% confidence bounds) } & \multicolumn{2}{|c|}{ Goodness of fit } \\
\hline$h$ & -99.57 & $(-108.5,-90.62)$ & $\overline{\text { SSE }}$ & 0.5143 \\
\hline$i$ & -0.1143 & $(-0.1273,-0.1013)$ & R-Square & 0.9997 \\
\hline$j$ & 39.25 & $(30.14,48.37)$ & Adjusted R-Square & 0.9997 \\
\hline & & & RMSE & 0.138 \\
\hline
\end{tabular}

Tab. 4. Coefficients and goodness of fit for fitted power equation (6).

smoother in the cases with higher numbers of runs. Picking the smoothest curve from the simulation with 500 runs, curve fitting was used to find the relevant relationship equations. During the process of curve fitting different functions were tried. Exponential and power curves were found to be the most accurate ones with acceptable goodness of fit levels. The exponential function resulted in (5). The coefficients of $d, e, f, g$, and the goodness of fittest results listed in Tab. 3 and the power function resulted in (6). Coefficients of $h, i, j$ and the goodness of fit levels are tabulated in Tab. 4.

$$
\begin{gathered}
R S S_{\text {total }}=d \times \exp (e \times N)+f \times \exp (g \times N) \\
R S S_{\text {total }}=h \times N^{i}+j
\end{gathered}
$$

Figures 9 and 10 show the average RSS over 500 runs and the fitting curves based on exponential and power curves. As can be seen, power function equation is simpler than the exponential function equation. Also, Tabs. 3 and 4 show that the goodness of fit value of (6) makes it better than the (5) for our application. Therefore, the power function (6) was chosen for calculation of the number of simultaneously transmitting nodes. In this equation $N$ is the number of simultaneously transmitting nodes and $R S S_{\text {total }}$ is the calculated total received signal at the reference receiving node.

\section{Evaluating Accuracy and Consistency of the Proposed Algorithm}

In this work scenarios were simulated with different number of nodes in random positions. A snapshot approach was used and each snapshot was a totally different VANET topology to compare with previous scenarios with the same number of nodes. This approach has the advantage of evaluating the most complicated scenarios. In real scenarios this

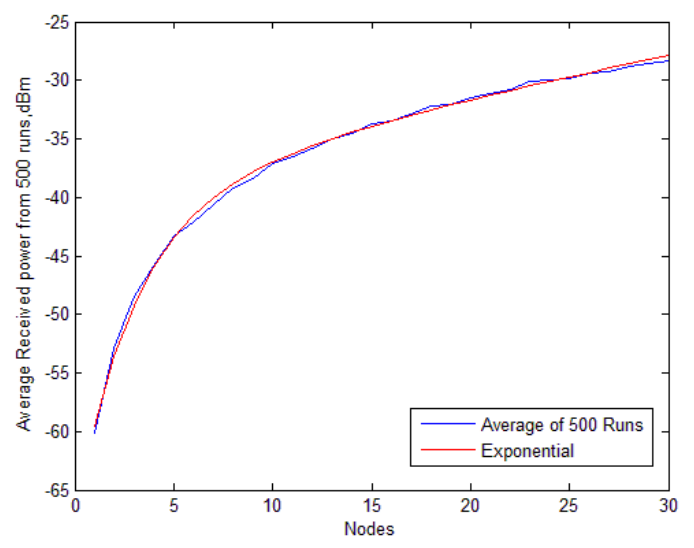

Fig. 9. Exponential curve fitting.

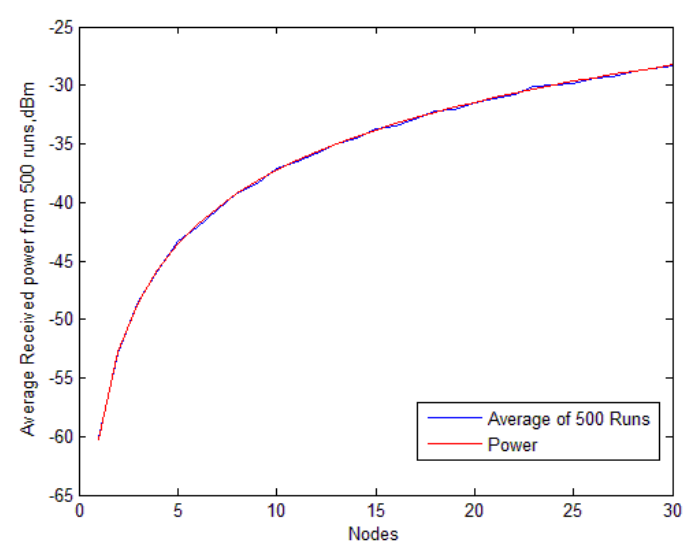

Fig. 10. Power curve fitting.

situation happens when the history of the network topology is not available for the nodes. This can be due to lack of beacon messages, or because the vehicle suddenly travels from a less dense network to a congested network and therefore, previous information is no longer valid. For such situations a node density estimation strategy which is independent of the history of the network topology makes the system more robust. Observations revealed that using only one snapshot and one RSS reading might not be adequate for 
an accurate node density estimation. Therefore, the results were collected again, this time in groups of 10 to 50 snapshots and $N$ was calculated based on the mean value of the collected RSSs in each group. This means, in the new approach, the receiving node will do the channel sensing for a number of times and make the decision about node density by using the average of the collected values. Figure 11 shows the standard deviation when the decision about node density estimation was made based on the collected RSSs for scenarios with 10, 20 and 50 readings at a time. The standard deviation reduces as a greater number of RSSs are collected for a decision. This approach is valid and practical for real scenarios in a saturated channel. In these situations transmitting nodes always have a packet to transmit and the state of the channel does not remain idle for a noticeable period of time. In addition, this system is being designed for working in the Control Channel of DSRC spectrum which is dedicated to safety messages. According to the standard for safety messages a MAC frame is a short frame of 512-1024 bytes in size. Therefore, listening to the channel and collecting a group of RSS readings, in the case of transmission of short packets for estimating node density, is not enforcing any considerable delay on the system. This system can be set to a certain number of RSS reads based on the environment and network situation. The situation of the network and the environment can be obtained from GPS which is available to all vehicles in VANETs. In addition, in order to avoid errors in this algorithm, a standard deviation of $\pm 10 \%$ was added to the calculated $N$ from (6). Thus, the corrected $N$ will be representative of a larger probability.

\section{Comparison between Different Approaches}

This section contains a brief feature-based comparison between performances of our solution with other node density estimation solutions in the literature. As mentioned in Section 2, some solutions rely on the information that is exchanged through beacon messages, [2, 3, 4]. These solutions would not perform well in case of broadcast storm where probability of missing beacon messages is high due to collision. However, the strength of our proposal is about its ability to perform well in any situation and even most strongly in presence of collision. In another approach, estimation of node density has been done based on reception of beacon messages [5]. This solution uses the missing beacon messages, assuming that the packet drop is due to collision. This cannot be reliable since, in wireless networks loss of beacon messages is not always due to collision and other factors such as radio propagation problem or network topology changes might cause beacon loss. Our solution instead, focuses on the received signal strength which is an accurate indication of number of transmitting nodes. Finally, in the last category, the solutions estimate node density based on the traffic flow [6], [7]. These solutions cannot perform well

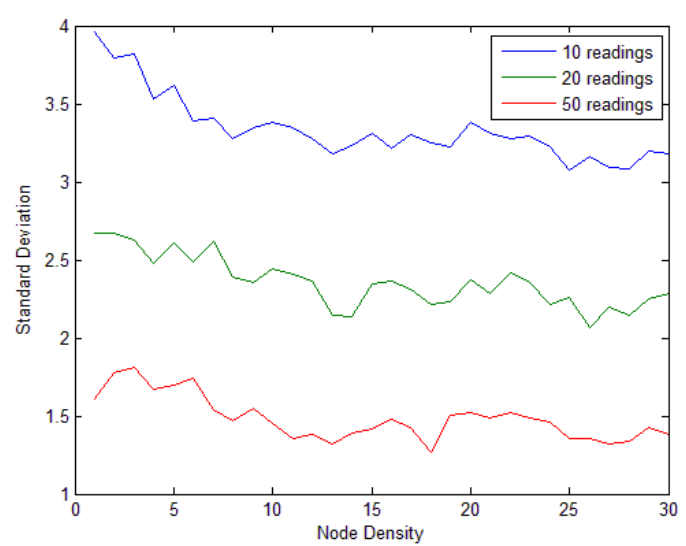

Fig. 11. Standard deviation of the node density estimation in different number of readings.

in some situations especially, in sparse networks. Moreover, in many cases changes of speed and acceleration of vehicles might be due to different reasons other than being in highly congested traffic especially, in urban areas. On a separate view, none of the solutions that are independent from the exchanged information between vehicles can estimate an exact node density range. Instead, they estimate a level of density that can be compared with a threshold value for specific adaptation solution. Our proposal not only performs independently from exchanged information between vehicles, it also calculates and estimates node density as an exact value which can be used for any application that requires an accurate estimation of node density. This solution is able to perform well in highly saturated networks with the high chance of collision and can be adaptive to different environments.

\section{Conclusion and Future Work}

This paper proposes a novel solution to estimate node density using a discrete event simulator in parallel with a physical layer based model. Using this method, every individual node is able to monitor its channel and record several consecutive RSS values. The average value of collected RSSs is used as a parameter to calculate the number of simultaneously transmitting nodes using the inverse of (6). Then the estimated number of simultaneously transmitting nodes is used in the inverse of (1), which provides the relation between node density and number of successful nodes to transmit simultaneously.

Since there are many VANET protocols affected by the node density, this proposal can be used by any V-2-V communication protocol that depends on traffic conditions. The broadcast storm problem is one situation that can be addressed using an accurate node density estimation technique. By detecting the problem in advance, the predicted problem can be reported to the higher layers and the system can apply required adaptive collision avoidance techniques to prevent the problem. 
To the best of our knowledge, this work is the first node density detection mechanism proposed independent of beacon messages based on physical layer parameters. In the future, we are going to work towards making our solution adaptive to fast variation of network topology and the changes of the environments from highway to urban environments and vice versa. Also, defining the exact required number of consecutive RSS readings for an accurate decision on node density for different environments could be addressed. Using the proposed technique in combination with a reliable adaptive cross-layer solution would address the broadcast storm problem.

\section{References}

[1] TSENG, Y., NI, S., CHEN, Y., SHEU, J.-P. The broadcast storm problem in a mobile ad hoc network. Wireless Networks, 2002, vol. 8, no. $2-3$, p. $153-167$.

[2] PANICHPAPIBOON, S., PATTARA-ATIKOM, W. Evaluation of a neighbor-based vehicle density estimation scheme. In 8th International Conference on ITS Telecommunications 2008, p. 294-298.

[3] JERBI, M., SENOUCI, S-M., RASHEED, T., GHAMRIDOUDANE, Y. An infrastructure-free traffic information system for vehicular networks. In IEEE 66th Vehicular Technology Conference (VTC 2007-Fall). Baltimore (MD, USA), 2007, p. 2086-2090. DOI: 10.1109/VETECF.2007.438

[4] WISCHOFF, L., EBNER, A., ROHLING, H., LOTT, M., HALFMANN, R. SOTIS-a self-organizing traffic information system. In The 57th IEEE Semiannual Vehicular Technology Conference (VTC 2003-Spring). 2003, vol. 4, p. 2442-2446. DOI: 10.1109/VETECS.2003.1208829

[5] BALON, N., GUO, J. Increasing broadcast reliability in vehicular ad-hoc networks. In Proceedings of the 3rd International Workshop on Vehicular Ad Hoc Networks (VANET). Los Angeles (CA, USA), 2006, p. 104-105. DOI: 10.1145/1161064.1161088

[6] ARTIMY, M. Local density estimation and dynamic transmissionrange assignment in vehicular ad-hoc networks. IEEE Transactions on Intelligent Transportation Systems, 2007, vol. 8, no. 3, p. 400412. DOI: $10.1109 /$ TITS.2007.895290
[7] SHIRANI, R., HENDESSI, F., GULLIVER, T. A. Store-carryforward message dissemination in vehicular ad-hoc networks with local density estimation. In 2009 IEEE 70th Vehicular Technology Conference (VTC 2009-Fall). Anchorage (AK, USA), 2009, p. 1-6. DOI: 10.1109/VETECF.2009.5379108

[8] GAST, M. 802.11 Wireless Networks: The Definitive Guide. O'Reilly Media, Inc., 2005.

[9] ALEXANDER, P., HALEY, D., GRANT, A. Cooperative intelligent transport systems: $5.9-\mathrm{GHz}$ field trials. Proceedings of the IEEE, 2011, vol. 99, no. 7, p. 1213-1235. DOI: 10.1109/JPROC.2011.2105230

[10] MOLISCH, A. F. Wireless Communications, vol. 15. John Wiley \& Sons, 2010.

[11] GOZALVEZ, J., SEPULCRE, M., BAUZA, R., Impact of the Radio Channel Modelling on the Performance of VANET Communication Protocols. Telecommunication Systems, 2012, vol. 50, no. 3, p. 149167.

[12] MOLISH, A. F., TUFVESSON, F., KAREDAL, J., MECKLENBRAUKER, C. Propagation aspects of vehicle-to-vehicle communications - an overview. IEEE Radio and Wireless Symposium (RWS). San Diego (CA, USA), 2009, p. 179-182. DOI: 10.1109/RWS.2009.4957315

[13] FONTAN, F. P., ESPINEIRA, P. M. Modelling the Wireless Propagation Channel: A Simulation Approach with MATLAB, vol. 5. John Wiley \& Sons, 2008.

[14] MOLISCH, A. F., TUFVESSON, F., KAREDAL, J., MECKLENBRAUKER, C. A survey on vehicle-to-vehicle propagation channels. IEEE Wireless Communications, 2009, vol. 16, no. 6, p. 12-22. DOI: 10.1109/MWC.2009.5361174

[15] ABBAS, T., SJOBERG, K., KAREDAL, J., TUFVESSON, F. A measurement based shadow fading model for vehicle-to-vehicle network simulations. Hindawi International Journal of Antennas and Propagation. Submitted. Preprint arXiv:1203.3370, 2012.

[16] LIN CHENG, HENTY, B. E., STANCIL, D. D., FAN BAI, MUDALIGE, P. Mobile vehicle-to-vehicle narrow-band channel measurement and characterization of the $5.9 \mathrm{ghz}$ dedicated short range communication (DSRC) frequency band. IEEE Journal on Selected Areas in Communications, 2007, vol. 25, no. 8, p. 1501-1516. DOI: 10.1109/JSAC.2007.071002. 